\title{
Neutral particles identification at LHCb
}

\author{
Boris Quintana*t \\ LPC, Clermont-Ferrand, France \\ E-mail: boris.quintana@cern. ch
}

\begin{abstract}
Important analyses of the core LHCb physics program rely on calorimetry to identify photons, high-energy neutral pions and electrons. For this purpose, the LHCb calorimeter system is composed of a scintillating pad plane, a preshower detector, an electromagnetic and a hadronic sampling calorimeters. The interaction of a given particle in these detectors leaves a specific signature. This is exploited for particle identification (PID) by combining calorimeters and tracking information into multivariate classifiers.

In this contribution, we focus on the identification of photons against high-energy neutral pions, electrons and hadronic backgrounds. Neutral particles reconstruction process at LHCb is succinctly explained and PID performances on Run 1 data is shown. Discrepancies with simulation predictions are then discussed, with special emphasis on the methods to correctly estimate PID cut efficiencies by means of large calibration samples of abundant beauty and charm decays to final states with photons or $\pi^{0}$. Finally, the technical aspects of the collection of these samples in Run 2 are presented.
\end{abstract}

Sixth Annual Conference on Large Hadron Collider Physics (LHCP2018)

4-9 June 2018

Bologna, Italy

\footnotetext{
* Speaker.

${ }^{\dagger}$ On behalf of the LHCb collaboration.
} 


\section{Introduction}

The ability to identify the nature of particles is one of the major requirements in a flavor physics experiment. At $\mathrm{LHCb}$, one challenging feature is the identification of neutrals. Important analyses of the core LHCb physics program rely on calorimetry to identify photons, high-energy neutral pions and electrons. For instance, a good $\gamma / \pi^{0}$ separation is an important prerequisite for the study of radiative decays such as $B_{s} \rightarrow \phi \gamma, B^{0} \rightarrow K^{*} \gamma, B^{ \pm} \rightarrow \phi K^{ \pm} \gamma$ and $\Lambda_{b} \rightarrow \Lambda^{0} \gamma$, to reject $\pi^{0}$ background contributions. Besides, this can be useful in the opposite case, rejecting photons when studying B decay modes with a $\pi^{0}$ in the final state, as in $B_{s} \rightarrow K K \pi^{0}$ and $B_{s} \rightarrow K \pi \pi^{0}$.

\section{Reconstruction of neutral particles}

The calorimeter system of LHCb [1] is composed of a Scintillating Pad Detector (SPD), a Preshower (PS), an Electromagnetic Calorimeter (ECAL) and a Hadronic Calorimeter (HCAL). The ECAL is based on the Shashlik technology and contains 6016 cells. In front of the ECAL, the PS is composed of 6016 tiles matching the geometry of the ECAL. The PS is placed after a lead sheet of 2.5 radiation length and is preceded by another layer of 6016 scintillator tiles, the SPD. The energy deposits of different particles in the calorimeters are displayed on Figure 1.

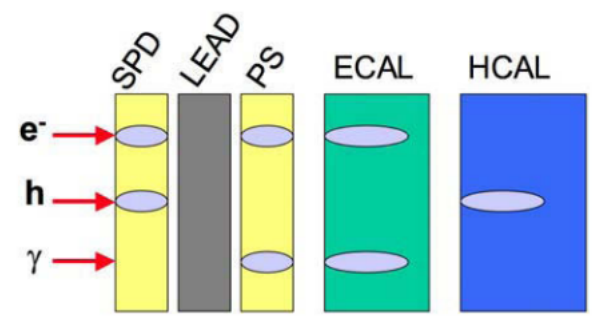

Figure 1: Energy deposited on the different parts of the calorimeter system by an electron, a hadron, and a photon

The reconstruction of electromagnetic showers process starts with the identification of the ECAL cell that has an excess in energy deposition, referred to as local maximum or seed cell, compared to all its direct neighbours (3-9 cells). These cells are selected only if the transverse energy is larger than $50 \mathrm{MeV}$. The identified cell will originate a cluster according to the clusterisation procedure adopted by the Cellular Automaton algorithm [2]. The selection of neutral clusters is performed using an anti-matching technique with reconstructed tracks : reconstructed tracks in the event are extrapolated to the calorimeter and then all-to-all matching with the reconstructed clusters is performed.

Around $88 \%$ of the photons detected by the calorimeter originate from $\pi^{0}$ decays. The signature in the ECAL depends on its kinematics, the higher the momentum of the $\pi^{0}$ is the closer the two photons are at the entry of the calorimeter. These two photons can then either produce two separated clusters or share a single cluster in which their individual signals are not clearly distinguishable. 
The $\pi^{0}$ are classified as resolved $\pi^{0}$ in the former case and as merged $\pi^{0}$ in the latter one, for which the transverse momentum spectrum starts around $2 \mathrm{GeV} / \mathrm{c}$. To reconstruct resolved $\pi^{0}$, photons are paired and their invariant mass, $\mathrm{m}_{\gamma \gamma}$, is compared with the $\pi^{0}$ mass : $\mathrm{m}_{\gamma \gamma}$ is required to be in the range 105 to $165 \mathrm{MeV} / \mathrm{c}^{2}$. To reconstruct merged $\pi^{0}$, each electromagnetic cluster is split in two subclusters defined from the two most energetic cells in the cluster. After the preparation of the two photon subclusters, a specific creteria relying on the energy in the cluster and the reconstructed merged $\pi^{0}$ mass is applied to identify the cluster as coming from a $\pi^{0}$. In $70 \%$ of cases, a merged $\pi^{0}$ can be misidentified as a single photon. Actually, the dominant background of the radiative decays corresponds to merged $\pi^{0}$, which amounts up to $50-60 \%$ of the background. Therefore the use of an efficient PID variable is crucial.

\section{Multivariate approaches to PID}

Particle identification (PID) algorithms are based on multivariate classifiers. They combine informations from different subdetectors, such as variables describing the track-cluster matching, or the shape of the neutral cluster, into a discriminant output. They are trained to separate photons signatures from hadrons and electrons, which may have passed the neutral cluster selection, and high-energy $\pi^{0}$.

Three different Neural Networks (MultiLayer Perceptrons - MLP) are trained using Monte Carlo simulation of either non-electromagnetic particles, electrons from $B \rightarrow K^{*} e e$ simulation, or decays with a $\pi^{0}$ such as $B^{0} \rightarrow K^{+} \pi^{-} \pi^{0}$, and where the hadron/electron $/ \pi^{0}$ is reconstructed as a photon. The three corresponding output variables are named respectively IsNotH, IsNotE and IsPhoton [3]. The PID variables distributions for both signal(true photons) and background(h/e/ $\pi^{0}$ ) are displayed on Figure 2.

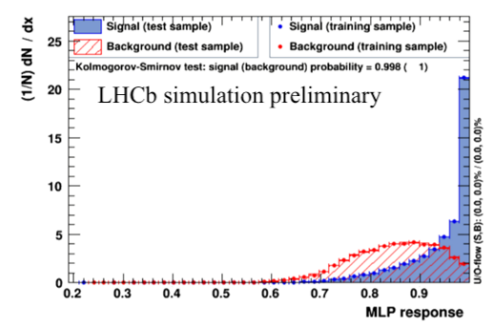

(a) IsNotE

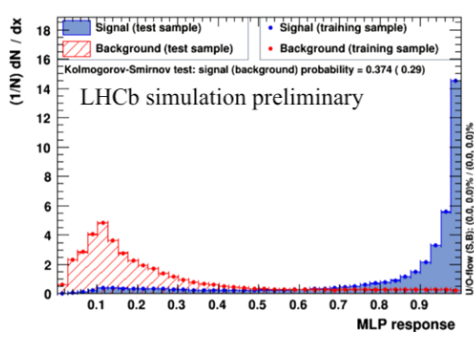

(b) IsNotH

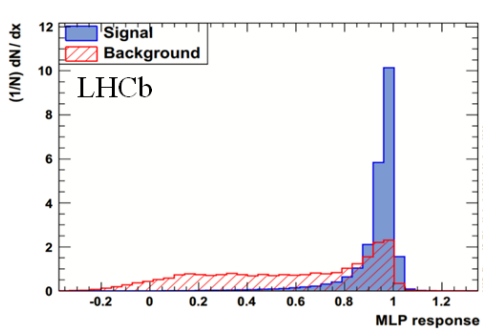

(c) IsPhoton

Figure 2: Output distributions of the neutral PID Neural Networks IsNotE, IsNotH and IsPhoton.

Due to MC/data discrepancies in the input variables used to build the $\gamma / \pi^{0}$ separation variable, discrepancies are also expected in the output of the method. It can be seen on Figure 3 where the photon efficiency versus $\pi^{0}$ rejection for both data and MC is shown.

In order to get a true estimate of the selection efficiency for a given cut on the IsPhoton variable, calibration samples from real data are needed. 


\section{Calibration samples}

In order to calibrate the performance of the IsPhoton variable, $B^{0} \rightarrow K^{*} \gamma$ reconstructed events are used as a calibration sample for photons and $D^{0} \rightarrow K \pi \pi^{0}$ events selected from $D^{*+} \rightarrow D^{0} \pi^{+}$ for merged $\pi^{0}$.

For the Run $1, B^{0} \rightarrow K^{*} \gamma$ sample takes into account the whole $B^{0} \rightarrow K^{*} \gamma$ data collected by the LHCb detector over the 2011-2012 period accounting for $3 \mathrm{fb}^{-1}$ of integrated luminosity. The $D^{0} \rightarrow K \pi \pi^{0}$ sample is obtained only using 2011 data. It has been selected from $D^{*+} \rightarrow D^{0} \pi^{+}$with a very tight mass cut $\left|M_{D^{*+}}-M_{D^{0}}\right|$, hence resulting in a very clean sample. In order to extract pure samples to use for the calibration, the invariant mass distribution is modeled and the sPlot technique [4] is then used to extract weights for the signal component so as to have background subtracted samples.

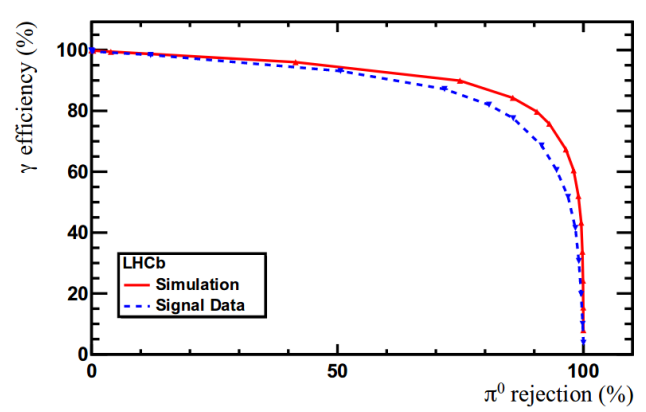

Figure 3: Photon selection efficiency as a function of $\pi^{0}$ rejection for data and MC simulation.

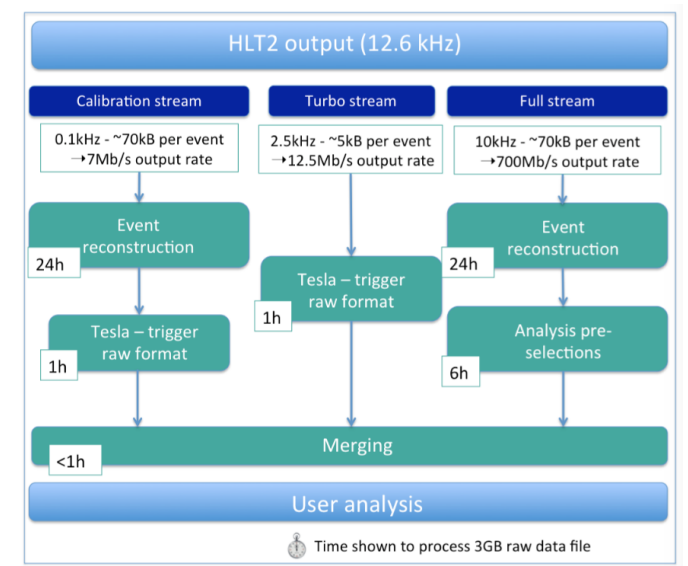

Figure 4: Workflow of the Calibration stream for Run 2.

For the Run 2, thanks to the real-time detector alignment and calibration, selected exclusive decays with both online (Turbo) and offline (Full) informations are available directly at the second High Level Trigger (HLT 2) with the TurCal stream (Turbo - Calibration [5], Figure 4). This feature allows to select bigger calibration samples with respect to Run 1, improving also the phase-space coverage adding new decay modes (Table 1). The preliminary mass fits for the $B^{0} \rightarrow K^{*} \gamma$ and $D^{0} \rightarrow K \pi \pi^{0}$ Run2 samples are shown in Figure 5 .

\begin{tabular}{c|c|c}
\multicolumn{2}{c|}{$\gamma$ calibration modes } & $\pi^{0}$ calibration modes \\
\hline $\mathrm{D}^{+} \rightarrow\left(\eta^{\prime} \rightarrow \pi^{+} \pi^{-} \gamma\right) \pi^{+}$ & $\mathrm{B} \rightarrow K^{*} \gamma$ & $\mathrm{D}^{*+}(2010) \rightarrow\left(D^{0} \rightarrow K^{-} \pi^{+} \pi^{0}\right) \pi^{+}\left(\pi^{0}\right.$ resolved $)$ \\
$\mathrm{D}_{s}^{*+} \rightarrow\left(D_{s}^{+} \rightarrow K^{+} K^{-} \pi^{+}\right) \gamma$ & $\mathrm{B}_{s} \rightarrow \phi \gamma$ & $\mathrm{D}^{*+}(2010) \rightarrow\left(D^{0} \rightarrow K^{-} \pi^{+} \pi^{0}\right) \pi^{+}\left(\pi^{0}\right.$ merged $)$
\end{tabular}

Table 1: Run 2 calibration modes for $\gamma$ and $\pi^{0}$. 
Once the calibration samples are produced, the efficiency for a given cut in the samples is computed in each bins of variables that are correlated with the PID (e.g. $E_{T}$, pseudo-rapidity).

The MC samples can then either be reweighted to match the PID selected data, or resampled to correct the distribution of the PID variable.
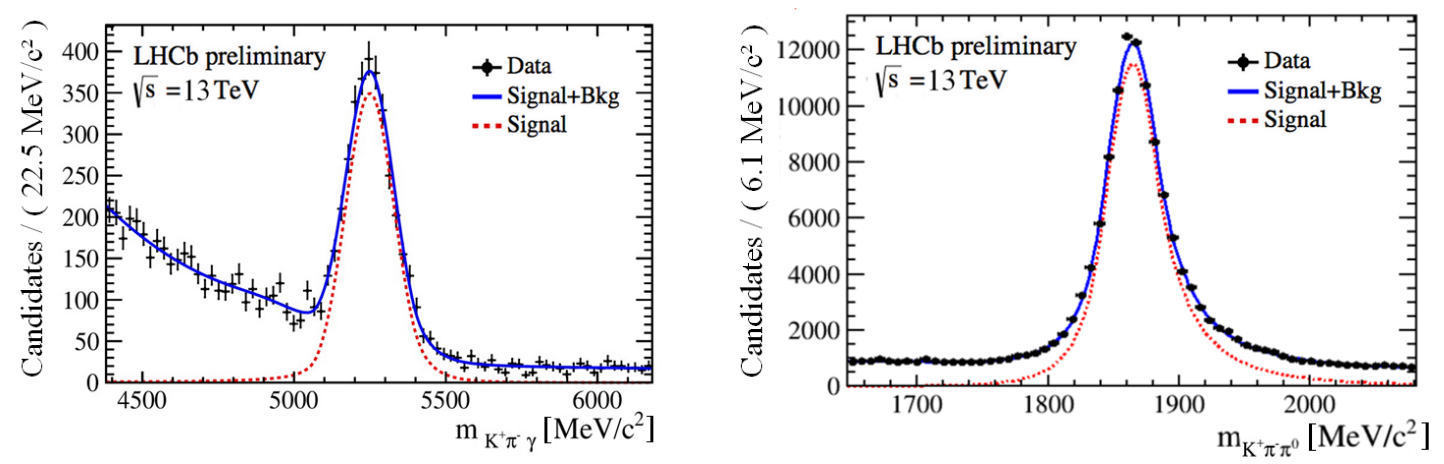

Figure 5: Fits to the $\mathrm{B}$ and $\mathrm{D}^{0}$ invariant mass in $B \rightarrow K^{*} \gamma$ (left) and $D^{0} \rightarrow K \pi \pi^{0}$ (right) calibration samples.

\section{Conclusion}

The separation between neutral particles signatures in the calorimeters is achieved with multivariate classifiers, using discriminant informations from the LHCb calorimeter sub-detectors, and gives good performances. However, as the simulated samples used for the Neural Network training does not represent the real data with full accuracy, discrepancies in the tools performance between data and MC are expected. From this comes the need for large samples of calibration data, to get a true estimate of PID requirements. While these samples are provided for photons and $\pi^{0}$ through a dedicated offline selection process for the Run 1, the Turbo-Calib stream implemented for the Run 2 allows a much more convenient online selection of large calibration samples. A dedicated tool is available to analysts to correct the $\gamma / \pi^{0}$ separation variable (IsPhoton) distribution in MC simulated samples, and work is on-going to propose a similar tool for the two others neutral PID variables.

\section{References}

[1] R. Aaij et al. [LHCb Collaboration], LHCb Detector Performance, Int. J. Mod. Phys. A 30 (2015) no.07, 1530022 [arXiv:1412.6352 [hep-ex]].

[2] V. Breton, N. Brun and P. Perret, A clustering algorithm for the LHCb electromagnetic calorimeter using a cellular automaton, LHCb-2001-123, CERN-LHCb-2001-123.

[3] M. Calvo Gomez et al., A tool for $\gamma / \pi^{0}$ separation at high energies Tech. Rep. 2252 LHCb-PUB-2015-016.

[4] M. Pivk and F. R. Le Diberder, SPlot: A Statistical tool to unfold data distributions, Nucl. Instrum. Meth. A 555 (2005) 356 [physics/0402083 [physics.data-an]].

[5] R. Aaij et al. [LHCb Collaboration], Tesla : an application for real-time data analysis in High Energy Physics, Comput. Phys. Commun. 208 (2016) 35-42 